\title{
ADDITIVE DERIVATIONS OF NEST ALGEBRAS
}

\author{
HAN DEGUANG
}

(Communicated by Palle E. T. Jorgensen)

\begin{abstract}
In this paper we prove that every additive derivation of a nest algebra acting on an infinite-dimensional Hilbert space is inner. This extends the relative result for linear derivations of nest algebras.
\end{abstract}

\section{INTRODUCTION}

The study of additive derivations was initiated by Johnson and Sinclair in [6] where they proved the following result: Let $\mathscr{A}$ be a semisimple Banach algebra and $D: \mathscr{A} \rightarrow \mathscr{A}$ be an additive derivation. Then $\mathscr{A}$ contains a central idempotent $e$ such that $e \mathscr{A}$ and $(I-e) \mathscr{A}$ are invariant for $D, D_{(I-e) \mathscr{A}}$ is continuous, and $e \mathscr{A}$ is finite dimensional. This result implies that every additive derivation from $L(H)$ into $L(H)$ is inner, provided that $H$ is an infinite-dimensional Hilbert space. Motivated by this result, we consider the additive derivation problem on nest algebras.

First we recall some definitions. Let $L(H)$ be the algebra of all linear bounded operators on Hilbert space $H$. A nest $\mathcal{N}$ is a complete totally ordered family of selfadjoint projections on $H$ that contains 0 and $I$. The algebra alg $\mathscr{N}=\left\{T \in L(H): P^{\perp} T P=0\right.$ for all $\left.P \in \mathscr{N}\right\}$ is called the nest algebra with respect to the nest $\mathscr{N}$.

For a subalgebra $\mathscr{A}$ of $L(H)$, an additive (linear) map $D: \mathscr{A} \rightarrow L(H)$ is said to be an additive (linear) derivation if $D(A B)=A D(B)+D(A) B$ holds for all $A, B \in \mathscr{A}$. D is said to be inner if there exists an operator $T$ in $L(H)$ such that $D(A)=A T-T A$ holds for all $A$ in $\mathscr{A}$.

It is well known that if $\mathscr{A}$ is a nest algebra on $H$, then every linear derivation from $\mathscr{A}$ into $L(H)$ is inner [2]. A natural question is: Is every additive derivation of a nest algebra inner? If $\operatorname{dim} H<\infty$, then there exist additive derivations of the nest algebra which are not inner (see [4, 7]). In [4] we proved that every additive derivation of a triangular operator algebra acting on an infinite-dimensional Hilbert space is inner. The purpose of this paper is to show that this is true for any nest algebra acting on an infinite-dimensional Hilbert space.

Received by the editors March 25, 1992.

1991 Mathematics Subject Classification. Primary 47D25, 47B47, 47D15.

Key words and phrases. Nest algebra, additive derivation, additive map, inner derivation.

Research partially supported by the NSF of China and the NSF of Shandong. 


\section{THE MAIN PART}

First we give some definitions and state some basic facts.

Let $K_{1}$ and $K_{2}$ be two linear spaces over $\mathbb{C}$. A map $T$ from $K_{1}$ into $K_{2}$ is called additive if $T(x+y)=T x+T y$ holds for all $x, y \in K_{1}$.

The following Lemma 2.1 is taken from [4].

Lemma 2.1. Let $\mathcal{N}$ be a nest in $L(H)$ and $D$ an additive derivation from alg $\mathscr{N}$ into $L(H)$. Then there exists an additive map $T$ from $K$ into $H$ such that $D(A)=A T-T A$ holds on $K$ for all $A \in \operatorname{alg} \mathcal{N}$, where $K=H$ if $I_{-} \leq I$ and $K=\bigcup\{P H: P \in \mathscr{N}$ and $P<I\}$ if $I_{-}=I$. Here $I_{-}=\bigvee\{P: P \in \mathscr{N}, P<I\}$.

Lemma 2.2. Let $\mathcal{N}$ be a nest in $L(H)$ and $D: \operatorname{alg} \mathcal{N} \rightarrow L(H)$ be a linear derivation. Assume that $T: K \rightarrow H$ is an additive map such that $D(A)=$ $A T-T A$ for all $A \in \operatorname{alg} \mathcal{N}$. Here $K$ is as in Lemma 2.1. Then $T$ is linear on $K$.

Proof. By Christensen's result [2], there exists an operator $S \in L(H)$ such that $D(A)=A S-S A$ holds for all $A \in \operatorname{alg} \mathcal{N}$. Thus $(T-S) A=A(T-S)$ on $K$ for all $A \in \operatorname{alg} \mathscr{N}$.

(1) If $I_{-}<I$, then we can choose $y \in\left(I-I_{-}\right) H$ such that $\|y\|=1$. For any $x \in H$, define $A \in \operatorname{alg} \mathcal{N}$ as

$$
A z=\langle z, y\rangle x, \quad z \in H .
$$

Then $(T-S) A y=A(T-S) y$, i.e., $(T-S) x=\langle(T-S) y, y\rangle x$. Since $x$ is arbitrary, we obtain that $(T-S)=\lambda I$ for some $\lambda \in \mathbb{C}$. Thus $T$ is linear on $H$.

(2) Suppose that $I_{-}=I$. Take $P_{n} \in \mathcal{N}$ such that $P_{n}<I$ and $P_{n} \uparrow I$ in the strong operator topology. Then $K=\bigcup P_{n} H$ is dense in $H$. For any $x \in K$, there is some $n$ such that $x \in P_{n} H$. Take $y \in\left(P_{n+1}-P_{n}\right) H$ such that $\|y\|=1$. Define $A \in \operatorname{alg} \mathscr{N}$ as

$$
A z=\langle z, y\rangle x, \quad z \in H .
$$

Since $(T-S) A y=A(T-S) y$, we obtain

$$
(T-S) x=\langle(T-S) y, y\rangle x ;
$$

thus $(T-S) x=\lambda_{x} x$ for some $\lambda_{x} \in \mathbb{C}$, which implies that $T-S=\lambda I$ on $K$ for some $\lambda \in \mathbb{C}$. Therefore, $T$ is linear on $K$.

Remark. From the above proof, we have that, if $D$ and $T$ satisfy the conditions in the lemma, then $T$ can be extended uniquely to an operator $T \in L(H)$ such that $D(A)=A T-T A$ for all $A \in \operatorname{alg} \mathscr{N}$. Thus we may suppose that $T \in L(H)$.

Let $\mathscr{N}$ be a nest in $L(H)$ and $P, Q \in \mathscr{N}$ such that $Q<P<I$. Define $\widetilde{D}(R A R)=R D(R A R) R$ for all $A \in \operatorname{alg} \mathcal{N}$, where $R=P-Q$ and $D$ is an additive derivation from alg $\mathcal{N}$ into $L(H)$. Then $\widetilde{D}$ can be regarded as an additive derivation from $R(\operatorname{alg} \mathcal{N}) R \subseteq L(R H)$ into $L(R H)$.

Lemma 2.3. Let $\mathcal{N}, P, Q, D$, and $\widetilde{D}$ be as above. If $\widetilde{D}$ is linear, then so is D.

Proof. From Lemma 2.1 there exists an additive map $T$ from $K$ into $H$ such that $D(A)=A T-T A$ holds on $K$ for all $A \in \operatorname{alg} \mathscr{N}$. 
It is a routine exercise that $T$ can be decomposed as $T=\left(T_{i j}\right)_{3 \times 3}$ with respect to $K=Q H+R H+P^{\perp} H$. The additive mappings $T_{13}: P^{\perp} H \rightarrow Q H$, $T_{23}: P^{\perp} H \rightarrow R H$, and $T_{33}: P^{\perp} H \rightarrow P^{\perp} H$ are defined on a dense subspace $M \subseteq P^{\perp} H$, where $M=\bigcup\{(E-P) H: E \in \mathscr{N}, P<E<I\}$ when $I_{-}=I$ and $M=P^{\perp} H$ when $I_{-}<I$.

Let

$$
B=\left[\begin{array}{ccc}
0 & 0 & 0 \\
0 & R A R & 0 \\
0 & 0 & 0
\end{array}\right], \quad A \in \operatorname{alg} \mathscr{N} .
$$

Then $B \in \operatorname{alg} \mathscr{N}$, and $\widetilde{D}(R A R)=R D(R B R) R=R A R T_{22}-T_{22} R A R$ for all $A \in \operatorname{alg} \mathscr{N}$. Since $\widetilde{D}$ is linear, by Lemma 2.2 we have that $T_{22}$ is linear on a dense subspace $N$ of $R H$.

Let

$$
A=\left[\begin{array}{lll}
I & 0 & 0 \\
0 & 0 & 0 \\
0 & 0 & 0
\end{array}\right],\left[\begin{array}{lll}
0 & 0 & 0 \\
0 & I & 0 \\
0 & 0 & 0
\end{array}\right],\left[\begin{array}{lll}
0 & 0 & 0 \\
0 & 0 & 0 \\
0 & 0 & I
\end{array}\right]
$$

respectively. Since $D(A)=A T-T A$ on $K$ and $D(A) \in L(H)$, we can obtain that $T_{i j}$ is linear when $i \neq j$.

Let

$$
A=\left[\begin{array}{lll}
0 & C & 0 \\
0 & 0 & 0 \\
0 & 0 & 0
\end{array}\right], \quad C \in L(R H, Q H) .
$$

Then $A \in \operatorname{alg} \mathscr{N}$, and

$$
D(A)=A T-T A=\left[\begin{array}{ccc}
C T_{21} & C T_{22}-T_{11} C & C T_{23} \\
0 & -T_{21} C & 0 \\
0 & -T_{31} C & 0
\end{array}\right]
$$

holds on $K$. Since $D(A) \in L(H)$, we have that $C T_{22}-T_{11} C$ is linear. For any $x \in Q H$ and $t \in \mathbb{C}$, take $y \in N$ such that $\|y\|=1$. Let $C z=\langle z, y\rangle x, z \in$ $R H$. Then, from $\left(C T_{22}-T_{11} C\right) t y=t\left(C T_{22}-T_{11} C\right) y$ and $C T_{22} t y=t C T_{22} y$, it follows that $T_{11} t x=T_{11} C t y=t T_{11} C y=t T_{11} x$. Thus, $T_{11}$ is linear.

Now we show that $T_{33}$ is linear. Let

$$
A=\left[\begin{array}{lll}
0 & 0 & C \\
0 & 0 & 0 \\
0 & 0 & 0
\end{array}\right], \quad C \in L\left(P^{\perp} H, Q H\right) .
$$

Then $A \in \operatorname{Alg} \mathscr{N}$. Since $D(A) \in L(H)$ and $D(A)=A T-T A$ on $K$, we have that $C T_{33}-T_{11} C$ is linear on $M$; thus, $C T_{33}$ is linear on $M$. Fix any $x \in M$ and $t \in \mathbb{C}$. For any $y \in P^{\perp} H$, define $C z=\langle z, y\rangle u, z \in P^{\perp} H$, where $u$ is a fixed element in $Q H$ such that $\|u\|=1$. Then

$$
C T_{33} t x=\left\langle T_{33} t x, y\right\rangle \text {. }
$$

On the other hand,

$$
C T_{33} t x=t C T_{33} x=\left\langle t T_{33} x, y\right\rangle .
$$

Thus $\left\langle T_{33} t x, y\right\rangle=\left\langle t T_{33} x, y\right\rangle$. Since $y$ is arbitrary in $P^{\perp} H$, we obtain that $T_{33} t x=t T_{33} x$. Hence $T_{33}$ is linear on $M$. 
Now we have proven that $T$ is linear on a dense linear manifold $\widetilde{K}$ of $H$. For any $t \in \mathbb{C}$ and $x \in H$, take $x_{n} \in \widetilde{K}$ such that $x_{n} \rightarrow x$. Then

$$
D(t I) x_{n}=(t I) T x_{n}-T(t I) x_{n}=0 .
$$

Therefore, $D(t I) x=0$, which implies that

$$
D(t I)=0=t D(I) .
$$

For any $A \in \operatorname{alg} \mathscr{N}$ and $t \in \mathbb{C}$,

$$
D(t A)=t I D(A)+D(t I) A=t D(A) .
$$

Thus $D$ is linear, and hence $D$ is inner.

Lemma 2.4. Let $\mathcal{N}$ be a nest in $L(H)$ and $D$ an additive derivation from $\operatorname{alg} \mathscr{N}$ into $L(H)$. If $D$ is not inner, then for any $M>0$ there exists $t \in \mathbb{C}$ such that $|t|<1$ and $\|D(t I)\| \geq M$.

Proof. Since $D$ is not inner, $D$ is not linear. It is easy to verify that $D(t I)$ is not linear in $t$, which implies that there is a continuous functional $g$ on $L(H)$ such that $\|g\|=1$ and $g(D(t I))$ is not linear in $t$. Let $f(t)=g(D(t I))$. Then $f$ is additive and is not continuous. By the result in [1], there exists $t \in \mathbb{C}$ such that $|t|<1$ and $|f(t)| \geq M$. Thus $\|D(t I)\| \geq|g(D(t I))| \geq M$.

Theorem 2.5. Let $\mathscr{N}$ be a nest acting on an infinite-dimensional Hilbert space $H$. Then every additive derivation from $\operatorname{alg} \mathscr{N}$ into $L(H)$ is inner.

Proof. $\mathscr{N}$ must satisfy one of the following three conditions: [4].

(1) $\mathscr{N}$ is a finite nest. Then the conclusion follows from Proposition 2.4 in

(2) There exist $P_{n} \in \mathcal{N}$ such that $P_{n}<P_{n+1}, n=1,2, \ldots$ Let $R_{n}=$ $P_{n}-P_{n-1}$, and define

$$
D_{n}\left(R_{n} A R_{n}\right)=R_{n} D\left(R_{n} A R_{n}\right) R_{n}, \quad A \in \operatorname{alg} \mathcal{N} .
$$

Then $D_{n}$ can be regarded as an additive derivation from $R_{n}(\operatorname{alg} \mathscr{N}) R_{n}$ into $L\left(R_{n} H\right)$.

We claim that there exists $n$ such that $D_{n}$ is linear, and thus by Lemma 2.3 $D$ is inner.

In fact, if every $D_{n}$ is not inner, then by Lemma 2.4 there exist $t_{n} \in \mathbb{C}$ such that $\left|t_{n}\right|<1$ and $\left\|D_{n}\left(t_{n} R_{n}\right)\right\| \geq n+2\left\|R_{n} D\left(R_{n}\right) R_{n}\right\|=n, n=1,2, \ldots$.

Let $S=\sum_{n=1}^{\infty} t_{n} R_{n}$. Then $S \in \operatorname{alg} \mathcal{N}$ and $R_{n} S=S R_{n}=t_{n} R_{n}$. Since $R_{n} D\left(R_{n} S R_{n}\right) R_{n}=R_{n} D\left(t_{n} R_{n}\right) R_{n}=D_{n}\left(t_{n} R_{n}\right)$ and

$$
\begin{aligned}
R_{n} D\left(R_{n} S R_{n}\right) R_{n} & =R_{n} D\left(S R_{n}\right) R_{n}+R_{n} D\left(R_{n}\right) S R_{n} \\
& =R_{n} D(S) R_{n}+R_{n} S D\left(R_{n}\right) R_{n}+R_{n} D\left(R_{n}\right) S R_{n} \\
& =R_{n} D(S) R_{n}+2 t_{n} R_{n} D\left(R_{n}\right) R_{n}=R_{n} D(S) R_{n},
\end{aligned}
$$

we have that

$$
\left\|R_{n} D(S) R_{n}\right\| \geq\left\|D_{n}\left(t_{n} R_{n}\right)\right\|-2\left|t_{n}\right|\left\|R_{n} D\left(R_{n}\right) R_{n}\right\| \geq n, \quad n=1,2, \ldots
$$

This contradicts the fact that

$$
\left\|R_{n} D(S) R_{n}\right\| \leq\|D(S)\| .
$$

(3) There exists a sequence $\left\{Q_{n}\right\} \subset \mathcal{N}$ such that $Q_{n+1}<Q_{n}, n=1,2, \ldots$ By considering $(\operatorname{alg} \mathscr{N})^{*}=\operatorname{alg} \mathscr{N}^{\perp}$ and $\bar{D}(A)=D\left(A^{*}\right)^{*}$ for all $A \in \operatorname{alg} \mathscr{N}^{\perp}$, where $\mathscr{N}^{\perp}=\left\{P: P^{\perp} \in \mathscr{N}\right\}$, from case (2) $\bar{D}$ is inner, and thus $D$ is inner. 
Corollary 2.6. Let $\mathscr{N}$ be a nest acting on an infinite-dimensional Hilbert space and $\mathscr{M}$ be an ultraweakly closed bimodule of $\mathscr{A}$ which contains the nest algebra. Then every additive derivation from $\mathscr{A}$ into $\mathscr{M}$ is inner.

Proof. This follows from Theorem 6 in [5] and Theorem 2.5.

\section{ACKNOWLEDGMENT}

The author expresses his thanks to the referee for several useful suggestions.

\section{REFERENCES}

1. J. Aczél, Lectures on functional equations and their applications, Academic Press, New York, 1966.

2. E. Christensen, Derivations of nest algebras, Math. Ann. 229 (1977), 155-161.

3. K. Davidson, Nest algebras, Pitman Res. Notes Math. Ser., vol. 191, Longman Sci. Tech, Harlow, 1988.

4. Han Deguang, Additive derivations of triangular operator algebras. (preprint)

5. $\ldots$ A note on the commutants of CSL algebras modulo bimodules, Proc. Amer. Math. Soc. 116 (1992), 707-709.

6. B. E. Johnson and A. M. Sinclair, Continuity of derivations and a problem of Kaplansky, Amer. J. Math. 90 (1968), 1067-1073.

7. P. Šemrl, Additive derivations of some operator algebras, Illinois J. Math. 35 (1991), 234-240.

Department of Mathematics, Qufu Normal University, Qufu, 273165, Shandong PeoPLE'S REPUBLIC OF CHINA 